\title{
INVERSIONS OF HERMITE SEMIGROUP
}

\author{
DU-WON BYUN
}

(Communicated by Palle E. T. Jorgensen)

\begin{abstract}
Let $\left\{e^{-c H} \mid c \geq 0\right\}$ be the Hermite semigroup on the real line $\mathbb{R}$. Then a representation is constructed for inversions of the semigroup, and it gives a representation of $e^{-c H}$ for $c<0$. Moreover, some characterizations of the domain in which, for $c<0, e^{-c H}$ is well defined are examined.
\end{abstract}

\section{INTRODUCTION}

We let $\mu$ be the Gaussian measure $\frac{1}{\sqrt{\pi}} e^{-x^{2}} d x$ on $\mathbb{R}$. Then the family of the normalized Hermite polynomials

$$
h_{n}(x)=\frac{1}{\sqrt{2^{n} n !}} e^{x^{2}} \frac{d^{n}}{d x^{n}} e^{-x^{2}}, \quad n=0,1,2, \ldots,
$$

is a complete orthonormal system in $\mathrm{L}^{2}(\mu)$. For any $f \in \mathrm{L}^{2}(\mu)$, let $f(x)=$ $\sum_{n=0}^{\infty} a_{n} h_{n}(x)$. If $c$ is nonnegative, then the series $\sum_{n=0}^{\infty} a_{n} e^{-c n} h_{n}(x)$ converges in $\mathrm{L}^{2}(\mu)$. Hence we can define the linear operator $e^{-c H}$ on $\mathrm{L}^{2}(\mu)$ by

$$
\left[e^{-c H} f\right](x)=\sum_{n=0}^{\infty} a_{n} e^{-c n} h_{n}(x),
$$

and the operator norm of $e^{-c H}$ is 1 . The Hermite semigroup on $\mathbb{R}$ means the family $\left\{e^{-c H} \mid c \geq 0\right\}$. More generally, for every complex number $c$ with $\Re c>0$ or $c=0$, we shall consider the operator $e^{-c H}$, which is examined in several papers (for instance, see [4, 10]). For $\Re c>0$, the operator $e^{-c H}$ is represented by

$$
\left[e^{-c H} f\right](x)=\frac{1}{\sqrt{\pi}} \int_{\mathbb{R}} p_{c}(x-\xi) f(\xi) e^{-\xi^{2}} d \xi, \quad f \in \mathrm{L}^{2}(\mu), x \in \mathbb{R},
$$

where the integral kernel

$$
p_{c}(x-\xi)=\sum_{n=0}^{\infty} e^{-c n} h_{n}(x) h_{n}(\xi)=\left(1-e^{-2 c}\right)^{-1 / 2} \exp \left\{\xi^{2}-\frac{\left(\xi-e^{-c} x\right)^{2}}{1-e^{-2 c}}\right\} .
$$

Received by the editors September 22, 1991.

1991 Mathematics Subject Classification. Primary 47D05; Secondary 30C40.

Key words and phrases. Analytic extension, entire function, Hermite polynomial, Hermite semigroup, inverse of operator, positive matrix, reproducing kernel Hilbert space. 
In this paper, for any $c$ with $\Re c>0$ we shall give a representation for the inverse of the operator $e^{-c H}$ in terms of integrals. Our argument for its representation is directly related to an extension of the operator $e^{-c H}$ for $\Re c<0$. When $\Re c<0$, it is obvious that the domain in which the operator $e^{-c H}$ is well defined is properly contained in $\mathrm{L}^{2}(\mu)$. We let $\mathscr{D}\left(e^{-c H}\right)=\left\{f \in \mathrm{L}^{2}(\mu) \mid e^{-c H} f \in \mathrm{L}^{2}(\mu)\right\}$. Then we give two characterizations of the members in $\mathscr{D}\left(e^{-c H}\right)$ in terms of analytic extensibility of members in $\mathrm{L}^{2}(\mu)$ as entire functions.

We shall use the general method for integral transforms ([8] or [9, p. 82]) and some ideas for best approximation oriented in [1] on reproducing kernel Hilbert spaces.

\section{INVERSE OF $e^{-c H}$}

For any fixed complex number $c$ with $\Re c>0$, applying the dominated convergence theorem and Morera's theorem to the integral representation (1.2), we see that every member in the range of the operator $e^{-c H}$ can be analytically extended to the complex plane $\mathbb{C}$. Hence we shall consider the operator $e^{-c H}$ as the linear operator of $\mathrm{L}^{2}(\mu)$ into an entire function space. Then, following the method of characterizing the ranges of integral transforms $([8]$ or $[9, p .82])$, we obtain

Theorem 1.1. For any $c$ with $\Re c>0$, the range of $\mathrm{L}^{2}(\mu)$ under the operator $e^{-c H}$ coincides with the Hilbert space consisting of entire functions with finite norms

$$
\begin{aligned}
\|g\|_{c}^{2}= & \frac{2|w|^{2}}{\pi \sqrt{1-|w|^{4}}} \\
& \cdot \iint_{\mathbb{C}}|g(z)|^{2} \exp \left\{-2|w|^{2}\left(\frac{x^{2}}{1+|w|^{2}}+\frac{y^{2}}{1-|w|^{2}}\right)\right\} d x d y
\end{aligned}
$$

where $z=x+i y$ and $w$ denotes $e^{-c}$. Moreover, the isometrical identity

$$
\left\|e^{-c H} f\right\|_{c}^{2}=\frac{1}{\sqrt{\pi}} \int_{\mathbb{R}}|f(x)|^{2} e^{-x^{2}} d x, \quad f \in \mathrm{L}^{2}(\mu),
$$

is valid.

Proof. For any pair $(z, u) \in \mathbb{C} \times \mathbb{C}$, we calculate the complex kernel form

$$
\begin{aligned}
K(z, \bar{u} ; c)= & \frac{1}{\sqrt{\pi}} \int_{\mathbb{R}} p_{c}(z-\xi) \overline{p_{c}(u-\xi)} e^{-\xi^{2}} d \xi \\
= & \frac{1}{\sqrt{\pi\left(1-w^{2}\right)\left(1-\bar{w}^{2}\right)}} \exp \left\{\frac{-w^{2} z^{2}}{1-w^{2}}\right\} \exp \left\{\frac{-\bar{w}^{2} \bar{u}^{2}}{1-\bar{w}^{2}}\right\} \\
& \cdot \int_{\mathbb{R}} \exp \left\{-\frac{1-|w|^{4}}{\left(1-w^{2}\right)\left(1-\bar{w}^{2}\right)} \xi^{2}\right. \\
& \left.\quad+\frac{2\left(1-\bar{w}^{2}\right) w z+2\left(1-w^{2}\right) \overline{w u}}{\left(1-w^{2}\right)\left(1-\bar{w}^{2}\right)} \xi\right\} \xi \\
= & \frac{1}{\sqrt{1-|w|^{4}}} \exp \left\{\frac{-|w|^{4} z^{2}}{1-|w|^{4}}\right\} \exp \left\{\frac{-|w|^{4} \bar{u}^{2}}{1-|w|^{4}}\right\} \exp \left\{\frac{2|w|^{2} z \bar{u}}{1-|w|^{4}}\right\} .
\end{aligned}
$$


Since $K(z, \bar{u} ; c)$ is a positive matrix on $\mathbb{C}$, it uniquely determines the reproducing kernel Hilbert space $H_{c}$ admitting the reproducing kernel $K(z, \bar{u} ; c)$. From [8] or [9, p. 82], the space $H_{c}$ is the range of $\mathrm{L}^{2}(\mu)$ under the operator $e^{-c H}$ and we have the isometrical identity

$$
\left\|e^{-c H} f\right\|_{H_{c}}^{2}=\frac{1}{\sqrt{\pi}} \int_{\mathbb{R}}|f(x)|^{2} e^{-x^{2}} d x, \quad f \in \mathrm{L}^{2}(\mu),
$$

because the family $\left\{p_{c}(z-\xi) \mid z \in \mathbb{C}\right\}$ is complete in $\mathrm{L}^{2}(\mu)$. Hence it is sufficient to prove that the members in $H_{c}$ are characterized as entire functions with finite norms (2.1). If $g \in H_{c}$, then $g$ is expressible in the form

$$
g(z)=\left(1-|w|^{4}\right)^{-1 / 2} \exp \left\{\frac{-|w|^{4} z^{2}}{1-|w|^{4}}\right\} g_{1}(z),
$$

where $z \in \mathbb{C}$ and $g_{1}$ is a member in the reproducing kernel Hilbert space $H_{\exp \left\{2|w|^{2} z \bar{u} /\left(1-|w|^{4}\right)\right\}}$ admitting the reproducing kernel $\exp \left\{\frac{2|w|^{2} z \bar{u}}{1-|w|^{4}}\right\}$. Moreover, the following isometrical identity holds:

$$
\|g\|_{H_{c}}^{2}=\left(1-|w|^{4}\right)^{-1 / 2}\left\|g_{1}\right\|_{H_{\exp \left\{2|w|^{2} z \bar{u} /\left(1-|w|^{4}\right)\right\}}^{2}} .
$$

For these contents, see [2, p. 358]. Meanwhile, referring to [3, p. 198], we have

$$
\left\|g_{1}\right\|_{H_{\exp \left\{2|w|^{2} z \bar{u} /\left(1-|w|^{4}\right)\right\}}^{2}}=\frac{2|w|^{2}}{\pi\left(1-|w|^{4}\right)} \iint_{\mathbb{C}}\left|g_{1}(z)\right|^{2} \exp \left\{-\frac{2|w|^{2}|z|^{2}}{1-|w|^{4}}\right\} d x d y .
$$

Therefore, from (2.3) and (2.4) our theorem is proved.

For two complex numbers $c_{1}, c_{2}$ with $\Re c_{1}>0$ and $\Re c_{2}>0$, we shall discuss a relation between $H_{c_{1}}$ and $H_{c_{2}}$. Put $g_{1}=e^{-c_{1} H} f$ and $g_{2}=e^{-c_{2} H} f$ for some $f \in \mathrm{L}^{2}(\mu)$. If $\Re c_{1}>\Re c_{2}$, then $g_{1}=e^{-c_{1} H} f=e^{-\left(c_{1}-c_{2}\right) H} e^{-c_{2} H} f=e^{-\left(c_{1}-c_{2}\right) H} g_{2}$, and so we can directly obtain a representation of $g_{1}$ in terms of $g_{2}$ by using the integral representation (1.2). However, if $\Re c_{1} \leq \Re c_{2}$, it is not obvious to represent $g_{1}$ in terms of $g_{2}$. Hence we are interested in this case. Furthermore, we shall establish the inverse formula of the integral transform (1.2).

Theorem 2.2. For $\Re c_{1}>0, \Re c_{2}>0$, and $f \in \mathrm{L}^{2}(\mu)$, let $g_{1}=e^{-c_{1} H} f$ and $g_{2}=e^{-c_{2} H} f$. Then $g_{1}$ is expressible in the form

$$
\begin{aligned}
& g_{1}(\xi)= \frac{2\left|w_{2}\right|^{2}}{\pi \sqrt{\left(1-\left|w_{2}\right|^{4}\right)\left(1-w_{1}^{2}{\overline{w_{2}}}^{2}\right)}} \exp \left\{\frac{-w_{1}^{2}{\overline{w_{2}}}^{2} \xi^{2}}{1-w_{1}^{2}{\overline{w_{2}}}^{2}}\right\} \\
& \cdot \iint_{\mathbb{C}} g_{2}(z) \exp \left\{\frac{w_{1} \bar{w}_{2} \bar{z}}{1-w_{1}^{2}{\overline{w_{2}}}^{2}}\left(2 \xi-w_{1} \overline{w_{2}} \bar{z}\right)\right. \\
&\left.-2\left|w_{2}\right|^{2}\left(\frac{x^{2}}{1+\left|w_{2}\right|^{2}}+\frac{y^{2}}{1-\left|w_{2}\right|^{2}}\right)\right\} d x d y,
\end{aligned}
$$

where $w_{1}=e^{-c_{1}}, w_{2}=e^{-c_{2}}$, and $g_{2}(z)$ is the analytic extension of $g_{2}$ to $\mathbb{C}$. 
Moreover, $f$ is given by

$$
\begin{aligned}
f(\xi)= & \text { 1. i.m. } \\
r \uparrow 1 & \frac{2\left|w_{2}\right|^{2}}{\pi \sqrt{\left(1-\left|w_{2}\right|^{4}\right)\left(1-r^{2}{\overline{w_{2}}}^{2}\right)}} \exp \left\{\frac{-r{\overline{w_{2}}}^{2} \xi^{2}}{1-r^{2}{\overline{w_{2}}}^{2}}\right\} \\
& \cdot \iint_{\mathbb{C}} g_{2}(z) \exp \left\{\frac{r \overline{w_{2}} \bar{z}}{1-r^{2} \bar{w}_{2}^{2}}\left(2 \xi-r \overline{w_{2}} \bar{z}\right)\right. \\
& \left.-2\left|w_{2}\right|^{2}\left(\frac{x^{2}}{1+\left|w_{2}\right|^{2}}+\frac{y^{2}}{1-\left|w_{2}\right|^{2}}\right)\right\} d x d y .
\end{aligned}
$$

The notation 1.i.m. means the $\mathrm{L}^{2}(\mu)$-convergence.

Proof. From the definition (1.1) of $e^{-c_{2} H}$ and the identity (2.5), the expression (2.6) is obvious. We assume that $T_{c_{1}}$ is the inverse operator of $e^{-c_{1} H}$ from $H_{c_{1}}$ to $\mathrm{L}^{2}(\mu)$. In addition, let $S_{c_{1}, c_{2}}$ be the linear operator of $H_{c_{1}}$ into $H_{c_{2}}$ defined by

$$
S_{c_{1}, c_{2}} g=e^{-c_{2} H} T_{c_{1}} g, \quad g \in H_{c_{1}} \text {. }
$$

Then we have $S_{c_{1}, c_{2}} g_{1}=g_{2}$. Since the operator $S_{c_{1}, c_{2}}$ is an isometry of $H_{c_{1}}$ onto $H_{c_{2}}$, the adjoint operator $S_{c_{1}, c_{2}}^{*}$ of $S_{c_{1}, c_{2}}$ is the inverse of it. Hence, for $\xi \in \mathbb{R}$ we get the representation

$$
g_{1}(\xi)=\left[S_{c_{1}, c_{2}}^{*} g_{2}\right](\xi)=\left(S_{c_{1}, c_{2}}^{*} g_{2}, K\left(\cdot, \xi ; c_{1}\right)\right)_{H_{c_{1}}}=\left(g_{2}, S_{c_{1}, c_{2}} K\left(\cdot, \xi ; c_{1}\right)\right)_{H_{c_{2}}} .
$$

Meanwhile, for $z \in \mathbb{C}$ the following is valid:

$$
\begin{aligned}
& {\left[S_{c_{1}, c_{2}} K\left(\cdot, \xi ; c_{1}\right)\right](z) } \\
&=\frac{1}{\sqrt{\pi}} \int_{\mathbb{R}} p_{c_{2}}(z-x) \overline{p_{c_{1}}(\xi-x)} e^{-x^{2}} d x \\
&=\frac{1}{\sqrt{\pi\left(1-\bar{w}_{1}^{2}\right)\left(1-w_{2}^{2}\right)}} \exp \left\{\frac{-\bar{w}_{1}^{2} \xi^{2}}{1-\bar{w}_{1}^{2}}\right\} \exp \left\{\frac{-w_{2}^{2} z^{2}}{1-w_{2}^{2}}\right\} \\
& \cdot \int_{\mathbb{R}} \exp \left\{-\frac{1-\bar{w}_{1}^{2} w_{2}^{2}}{\left(1-\bar{w}_{1}^{2}\right)\left(1-w_{2}^{2}\right)} x^{2}\right. \\
&= \frac{1}{\sqrt{1-\bar{w}_{1}^{2} w_{2}^{2}}} \exp \left\{\frac{\left.-w_{2}^{2}\right) \bar{w}_{1} \xi+2\left(1-\bar{w}_{1}^{2}\right) w_{2} z}{1-\bar{w}_{1}^{2} \bar{w}_{2}^{2} w}\right\} \\
& \cdot \exp \left\{\frac{-\bar{w}_{1}^{2} w_{2}^{2} z^{2}}{1-\bar{w}_{1}^{2} w_{2}^{2}}\right\} \exp \left\{\frac{2 \bar{w}_{1} w_{2} z \xi}{1-\bar{w}_{1}^{2} w_{2}^{2}}\right\} .
\end{aligned}
$$

Therefore, we obtain the identity (2.5).

In the expression (2.6), $r$ can be taken on a path in $\{0<|z|<1\}$ with the terminal point 1 , in general.

Remark. For any $n, h_{n}(z)$ denotes the analytic extension of $h_{n}(x)$ to $\mathbb{C}$. Then we see that, for $\Re c>0$, the family $\left\{e^{-c n} h_{n}(z) \mid n=0,1,2, \ldots\right\}$ is 
a complete orthonormal system in $H_{c}$. Hence, the expression $p_{c}(z-\xi)=$ $\sum_{n=0}^{\infty} e^{-c n} h_{n}(z) h_{n}(\xi)$ and Theorem 1.1 suggest the representation of $T_{c}$ in the form

$$
\left[T_{c} g\right](\xi)=\left(g, p_{c}(z-\xi)\right)_{H_{c}}, \quad g \in H_{c} .
$$

For fixed $\xi$, however, the integral in the right-hand side of (2.7) need not converge. In this case, in $[3$, p. $202 ; 7 ; 9$, p. 85] the method of taking some exhaustions of $\mathbb{C}$ was considered in a natural way. According to such a general method, we can obtain another representation of $(2.6)$ :

$$
\begin{aligned}
& f(\xi)=\underset{\sigma \rightarrow \infty}{\lim } \frac{2\left|w_{2}\right|^{2}}{\pi \sqrt{1-\left|w_{2}\right|^{4}}} \\
& \iint_{|z| \leq \sigma} g_{2}(z) \overline{p_{c_{2}}(z-\xi)} \exp \left\{-2\left|w_{2}\right|^{2}\left(\frac{x^{2}}{1+\left|w_{2}\right|^{2}}+\frac{y^{2}}{1-\left|w_{2}\right|^{2}}\right)\right\} d x d y \\
& =\underset{\sigma \rightarrow \infty}{\operatorname{i.m}} \frac{2\left|w_{2}\right|^{2}}{\pi \sqrt{\left(1-\left|w_{2}\right|^{4}\right)\left(1-\bar{w}_{2}^{2}\right)}} \\
& \cdot \iint_{|z| \leq \sigma} g_{2}(z) \exp \left\{\xi^{2}-\frac{\left(\xi-\overline{w_{2}} \bar{z}\right)^{2}}{1-\bar{w}_{2}^{2}}\right. \\
& \left.-2\left|w_{2}\right|^{2}\left(\frac{x^{2}}{1+\left|w_{2}\right|^{2}}+\frac{y^{2}}{1-\left|w_{2}\right|^{2}}\right)\right\} d x d y
\end{aligned}
$$

\section{EXTENSION OF $e^{-c H}$ FOR $\Re c<0$}

The inverse transform (2.6) means an extension of $e^{-c H}$ for $\Re c<0$ because $f(x)$ has the representation $\sum_{n=0}^{\infty} a_{n} e^{-\left(-c_{2}\right) n} h_{n}(x)$ in $\mathrm{L}^{2}(\mu)$ when $g_{2}(x)=$ $\sum_{n=0}^{\infty} a_{n} h_{n}(x)$. Hence, for any $c$ with $\Re c<0$, we define the linear operator $e^{-c H}$ in the form (1.1). Then, in the expression (2.6) replacing $w_{2}$ by $e^{c}$, we obtain the representation of $e^{-c H}$. However, since the expression (2.6) requires the analytic extension form of a member in $\mathrm{L}^{2}(\mu)$, we shall give its representation in terms of real variable.

For any fixed $c$ with $\Re c<0$, we first establish two characterizations of the members in $\mathscr{D}\left(e^{-c H}\right)$. Since the family $\left\{e^{c n} h_{n}(z) \mid n=0,1,2, \ldots\right\}$ is a complete orthonormal system in $H_{-c}$, for a given $f \in \mathrm{L}^{2}(\mu), f \in \mathscr{D}\left(e^{-c H}\right)$ if and only if $f$ is almost everywhere equal to the restriction of a member in $H_{-c}$ to $\mathbb{R}$ with respect to $\mu$. In this connection, we recall the interesting result in $[5$, p. 166]:

For any $\alpha>0$, let $h$ be an entire function satisfying $\iint_{\mathbb{C}}|h(z)|^{2} e^{-y^{2} / \alpha} d x d y<$ $\infty$. Then

$$
\frac{1}{\sqrt{\pi \alpha}} \iint_{\mathbb{C}}|h(z)|^{2} e^{-y^{2} / \alpha} d x d y=\sum_{n=0}^{\infty} \frac{\alpha^{n}}{n !} \int_{\mathbb{R}}\left|\frac{d^{n}}{d x^{n}} h(x)\right|^{2} d x .
$$

Conversely, if $h(x)$ is a $C^{\infty}$-function on $\mathbb{R}$ with a convergent sum in the righthand side of (3.1), then $h$ can be analytically extended to $\mathbb{C}$ and satisfies the identity (3.1).

This result will contribute to our result in the following way. 
Theorem 3.1. For any $c$ with $\Re c<0$, let $w=e^{c}$. If $f$ is a $C^{\infty}$-function in $\mathrm{L}^{2}(\mu)$, then the following are equivalent:

(1) $f$ is a member in $\mathscr{D}\left(e^{-c H}\right)$.

(2) The series

$$
\sum_{n=0}^{\infty} \frac{1}{n !}\left(\frac{1-|w|^{4}}{4|w|^{2}}\right)^{n} \int_{\mathbb{R}}\left|\frac{d^{n}}{d x^{n}}\left[f(x) \exp \left\{-\frac{|w|^{2} x^{2}}{1+|w|^{2}}\right\}\right]\right|^{2} d x
$$

converges.

(3) The integral

$$
\begin{aligned}
\iint_{\mathbb{C}}\left|\int_{\mathbb{R}} f(\xi) \exp \left\{\frac{\left(2|w|^{2} z-\xi\right) \xi}{1-|w|^{4}}\right\} d \xi\right|^{2} \\
\cdot \exp \left[\frac{-2|w|^{4}}{\left(1-|w|^{4}\right)\left(|w|^{8}+|w|^{4}+1\right)}\right. \\
\left.\cdot\left\{\left(|w|^{4}+|w|^{2}+1\right) x^{2}-\left(|w|^{4}-|w|^{2}+1\right) y^{2}\right\}\right] d x d y
\end{aligned}
$$

is finite.

Proof. If $f$ is the restriction of a member $\hat{f}$ in $H_{-c}$ to $\mathbb{R}$, then we have (3.4)

$$
\|\hat{f}\|_{-c}^{2}=\frac{2|w|^{2}}{\pi \sqrt{1-|w|^{4}}} \iint_{\mathbb{C}}\left|\hat{f}(z) \exp \left\{\frac{-|w|^{2} z^{2}}{1+|w|^{2}}\right\}\right|^{2} \exp \left\{\frac{-4|w|^{2} y^{2}}{1-|w|^{4}}\right\} d x d y .
$$

Therefore, from the identity (3.1) the two statements (1) and (2) are equivalent.

Next, we prove that $f$ is the restriction of a member in $H_{-c}$ to $\mathbb{R}$ if and only if $f$ satisfies the condition (3). For simplicity, let $T$ be the restriction operator to $\mathbb{R}$. Then $T$ is a bounded operator of $H_{-c}$ into $\mathrm{L}^{2}(\mu)$ (in fact, the operator norm $\|T\|$ is 1 ), and the adjoint operator $T^{*}$ of $T$ has the following representation:

$$
\left[T^{*} h\right](z)=(h, T K(\cdot, \bar{z} ;-c))_{\mathrm{L}^{2}(\mu)}, \quad z \in \mathbb{C},
$$

for all $h \in \mathrm{L}^{2}(\mu)$. Since the range $T\left(H_{-c}\right)$ is dense in $\mathrm{L}^{2}(\mu)$, the operator $T^{*}$ is one-to-one. Hence, $f$ can be extended as the member in $H_{-c}$ if and only if $T^{*} f$ is contained in the range $T^{*} T\left(H_{-c}\right)$. If $g$ is a member in $T^{*} T\left(H_{-c}\right)$, then $g$ is expressible in the form

$$
\begin{aligned}
g(z) & =\left(T^{*} T G, K(\cdot, \bar{z} ;-c)\right)_{H_{-c}} \\
& =\left(G, T^{*} T K(\cdot, \bar{z} ;-c)\right)_{H_{-c}}, \quad z \in \mathbb{C},
\end{aligned}
$$

for a member $G$ in $H_{-c}$ with $T^{*} T G=g$. Following a general method in [8] or $\left[9\right.$, p. 82], we shall give a characterization of the members in $T^{*} T\left(H_{-c}\right)$. From the representation (3.5) of $T^{*} T$, we calculate the kernel form

$$
\begin{aligned}
k(z, \bar{u} ; c) & =\left(T^{*} T K(\cdot, \bar{u} ;-c), T^{*} T K(\cdot, \bar{z} ;-c)\right)_{H_{-c}} \\
& =\left(T K(\cdot, \bar{u} ;-c), T T^{*} T K(\cdot, \bar{z} ;-c)\right)_{\mathrm{L}^{2}(\mu)} .
\end{aligned}
$$


Meanwhile, for all $z^{\prime} \in \mathbb{C}$ we have

$$
\begin{aligned}
& {\left[T^{*} T K(\cdot, \bar{z} ;-c)\right]\left(z^{\prime}\right) } \\
&=\left(T K(\cdot, \bar{z} ;-c), T K\left(\cdot, \bar{z}^{\prime} ;-c\right)\right)_{\mathrm{L}^{2}(\mu)} \\
&=\frac{1}{\left(1-|w|^{4}\right) \sqrt{\pi}} \exp \left\{\frac{-|w|^{4} z^{\prime 2}}{1-|w|^{4}}\right\} \exp \left\{\frac{-|w|^{4} \bar{z}^{2}}{1-|w|^{4}}\right\} \\
& \cdot \int_{\mathbf{R}} \exp \left\{-\frac{1+|w|^{4}}{1-|w|^{4}} \xi^{2}+\frac{2|w|^{2}\left(\bar{z}+z^{\prime}\right)}{1-|w|^{4}} \xi d \xi\right. \\
&= \frac{1}{\sqrt{1-|w|^{8}}} \exp \left\{\frac{-|w|^{8} z^{\prime 2}}{1-|w|^{8}}\right\} \exp \left\{\frac{-|w|^{8} \bar{z}^{2}}{1-|w|^{8}}\right\} \exp \left\{\frac{2|w|^{4} z^{\prime} \bar{z}}{1-|w|^{8}}\right\} .
\end{aligned}
$$

Hence, the desired kernel form is given by

$$
\begin{aligned}
k(z, \bar{u} ; c)= & \frac{1}{\sqrt{\pi\left(1-|w|^{4}\right)\left(1-|w|^{8}\right)}} \exp \left\{\frac{-|w|^{8} z^{2}}{1-|w|^{8}}\right\} \exp \left\{\frac{-|w|^{4} \bar{u}^{2}}{1-|w|^{4}}\right\} \\
& \cdot \int_{\mathbf{R}} \exp \left\{-\frac{|w|^{8}+|w|^{4}+1}{1-|w|^{8}} \xi^{2}+\left(\frac{2|w|^{4} z}{1-|w|^{8}}+\frac{2|w|^{2} \bar{u}}{1-|w|^{4}}\right) \xi\right\} d \xi \\
= & \frac{1}{\sqrt{\left(1-|w|^{4}\right)\left(|w|^{8}+|w|^{4}+1\right)}} \exp \left\{\frac{-|w|^{12} z^{2}}{\left(1-|w|^{4}\right)\left(|w|^{8}+|w|^{4}+1\right)}\right\} \\
& \cdot \exp \left\{\frac{-|w|^{12} \bar{u}^{2}}{\left(1-|w|^{4}\right)\left(|w|^{8}+|w|^{4}+1\right)}\right\} \\
& \cdot \exp \left\{\frac{2|w|^{6} z \bar{u}}{\left(1-|w|^{4}\right)\left(|w|^{8}+|w|^{4}+1\right)}\right\} .
\end{aligned}
$$

Then the range $T^{*} T\left(H_{-c}\right)$ coincides with the reproducing kernel Hilbert space $H_{k(c)}$ admitting the reproducing kernel $k(z, \bar{u} ; c)$, and $T^{*} T$ is an isometry of $H_{-c}$ onto $H_{k(c)}$. Let $H$ be the reproducing kernel Hilbert space determined by the positive matrix $\exp \left[2|w|^{6} z \bar{u} /\left\{\left(1-|w|^{4}\right)\left(|w|^{8}+|w|^{4}+1\right)\right\}\right]$. As in the proof of Theorem 1.2, we obtain the factorization

$$
\begin{aligned}
g(z)= & \frac{1}{\sqrt{\left(1-|w|^{4}\right)\left(|w|^{8}+|w|^{4}+1\right)}} \\
& \cdot \exp \left\{\frac{-|w|^{12} z^{2}}{\left(1-|w|^{4}\right)\left(|w|^{8}+|w|^{4}+1\right)}\right\} g_{1}(z)
\end{aligned}
$$

for an entire function $g_{1} \in H$. So it gives the norm identity

$$
\|g\|_{H_{k(c)}}^{2}=\frac{1}{\sqrt{\left(1-|w|^{4}\right)\left(|w|^{8}+|w|^{4}+1\right)}}\left\|g_{1}\right\|_{H}^{2},
$$

where

$$
\begin{aligned}
\left\|g_{1}\right\|_{H}^{2}= & \frac{2|w|^{6}}{\pi\left(1-|w|^{4}\right)\left(|w|^{8}+|w|^{4}+1\right)} \\
& \cdot \iint_{\mathbb{C}}\left|g_{1}(z)\right|^{2} \exp \left\{\frac{-2|w|^{6}|z|^{2}}{\left(1-|w|^{4}\right)\left(|w|^{8}+|w|^{4}+1\right)}\right\} d x d y .
\end{aligned}
$$


Note that $H$ is the totality of entire functions with finite norms (3.10). Hence, the space $H_{k(c)}$ consists of entire functions with finite norms

$$
\begin{aligned}
\|g\|_{H_{k(c)}}^{2}= & \frac{2|w|^{6}}{\pi \sqrt{\left(1-|w|^{4}\right)\left(|w|^{8}+|w|^{4}+1\right)}} \\
& \cdot \iint_{\mathbf{C}}|g(z)|^{2} \exp \left[\frac{-2|w|^{2}}{\left(1-|w|^{4}\right)\left(|w|^{8}+|w|^{4}+1\right)}\right. \\
& \left.\cdot\left\{\left(1-|w|^{6}\right) x^{2}+\left(1+|w|^{6}\right) y^{2}\right\}\right] d x d y .
\end{aligned}
$$

Since $T^{*} f$ is expressible in the form

$$
\left[T^{*} f\right](z)=\frac{1}{\sqrt{\pi\left(1-|w|^{4}\right)}} \exp \left\{\frac{-|w|^{4} z^{2}}{1-|w|^{4}}\right\} \int_{\mathbf{R}} f(\xi) \exp \left\{\frac{\left(2|w|^{2} z-\xi\right) \xi}{1-|w|^{4}}\right\} d \xi
$$

our claim has been proved.

When a $C^{\infty}$-function $f \in \mathrm{L}^{2}(\mu)$ satisfies one of three items in Theorem 3.1, we shall give the representation of the analytic extension $\hat{f}$ in terms of $f$. By the isometry $T^{*} T$ of $H_{-c}$ onto $H_{k(c)}$, we have

Theorem 3.2. Let $c, w$, and $f$ be as in Theorem 3.1. If $f$ satisfies one of three items in Theorem 3.1 and $\hat{f}$ denotes the analytic extension of $f$ to $\mathbb{C}$, then $\hat{f}$ is represented by

$$
\begin{gathered}
\hat{f}(z)=\frac{2|w|^{6}}{\pi^{3 / 2}\left(1-|w|^{4}\right) \sqrt{\left(1-|w|^{8}\right)\left(|w|^{8}+|w|^{4}+1\right)}} \exp \left\{\frac{-|w|^{8} z^{2}}{1-|w|^{8}}\right\} \\
\cdot \iint_{\mathbf{C}}\left[\int_{\mathbf{R}} f(\xi) \exp \left\{\frac{\left(2|w|^{2} Z-\xi\right) \xi}{1-|w|^{4}}\right\} d \xi\right] \\
\cdot \exp \left[\frac{-|w|^{4}}{1-|w|^{8}}\left(Z^{2}+|w|^{4} \bar{Z}^{2}-2 \bar{Z} z\right)\right. \\
\left.-\frac{2|w|^{6}\left\{\left(1-|w|^{6}\right) X^{2}+\left(1+|w|^{6}\right) Y^{2}\right\}}{\left(1-|w|^{4}\right)\left(|w|^{8}+|w|^{4}+1\right)}\right] d X d Y
\end{gathered}
$$

where $Z=X+i Y$.

Proof. Let $S$ be the adjoint operator of $T^{*} T$ from $H_{k(c)}$ to $H_{-c}$. Then, since $T^{*} T$ is an isometry of $H_{-c}$ onto $H_{k(c)}$, the operator $S$ is the inverse of $T^{*} T$. Hence, $T^{*} f=T^{*} T S T^{*} f$ and $f=T S T^{*} f$. Since

$$
\begin{aligned}
\hat{f}(z) & =\left[S T^{*} f\right](z)=\left(S T^{*} f, K(\cdot, \bar{z} ;-c)\right)_{H_{-c}} \\
& =\left(T^{*} f, T^{*} T K(\cdot, \bar{z} ;-c)\right)_{H_{k(c)}},
\end{aligned}
$$

from (3.7), (3.11), and (3.12) we obtain the desired representation (3.13).

\section{ACKNOWLEDGMENT}

The author wishes to thank Professor S. Saitoh for some valuable comments. 


\section{REFERENCES}

1. T. Ando and S. Saitoh, Restrictions of reproducing kernel Hilbert spaces to subsets, Preliminary reports, Suri Kaiseki Kenkyu Jo, Koukyu Roku 743 (1991), 164-187.

2. N. Aronszajn, Theory of reproducing kernels, Trans. Amer. Math. Soc. 68 (1950), 337-404.

3. V. Bargmann, On a Hilbert space of analytic functions and an associated integral transform. I, Comm. Pure Appl. Math. 14 (1961), 187-214.

4. W. Beckner, Inequalities in Fourier analysis, Ann. of Math. (2) 102 (1975), 159-182.

5. N. Hayashi and S. Saitoh, Analyticity and smoothing effect for the Schrödinger equation, Ann. Inst. Henri Poincare Phys. Théor. 52 (1990), 163-173.

6. E. Nelson, The free Markoff field, J. Funct. Anal. 12 (1973), 211-227.

7. S. Saitoh, Integral transforms in Hilbert spaces, Proc. Japan Acad. 58 (1982), 361-364.

8. (1983), 74-78.

9. __ Theory of reproducing kernels and its applications, Pitman Res. Notes Math. Ser., vol. 189, Longman Sci. Tech., Harlow, England, 1988.

10. F. B. Weissler, Two point inequalities, the Hermite semigroup, and the Gauss-Weierstrass semigroup, J. Funct. Anal. 32 (1979), 102-121.

Department of Mathematics, Faculty of Engineering, Gunma University, Kiryu 376, JAPAN

Current address: Department of Mathematics, Chung-ang University, 221 Huksok-dong, Tongjak-gu, Seoul 156-756, Korea 